\title{
eReader Interface Design for Page-turning
}

\author{
Nicholas Vanderschantz \\ Department of Computer Science \\ The University of Waikato \\ New Zealand \\ vtwoz@waikato.ac.nz
}

\author{
Claire Timpany \\ Department of Computer Science \\ The University of Waikato \\ New Zealand \\ ctimpany@waikato.ac.nz
}

\author{
Jiao Huang \\ Department of Computer Science \\ The University of Waikato \\ New Zealand \\ jh296@students.waikato.ac.nz
}

\begin{abstract}
There remain numerous unanswered questions regarding how to design eReaders that are effective for the range of reading purposes required by readers. This broad research area requires the attention of $\mathrm{HCl}$, design, and reading researchers alike. This paper begins to address the needs of readers when turning pages during reading in a digital book (eBook). Specifically, softwarebased page-turning features of eReading on a mobile device are investigated. A case study analysis of eReader software and hardware is presented and used to inform the design and testing of six page-turning methods for eReaders. Design recommendations for page-turning are provided.
\end{abstract}

\section{eReaders, eReading, Page-turning, User Preference, Interface Design}

\section{INTRODUCTION}

There is still scope for research regarding many aspects of designing effective eBooks, eReaders, and eReader software. Little is known about readers' preferences for different types of visual and interactive elements in reading eBooks. Elements of interface design such as content presentation, typography, illustration, and interaction are all features of eBooks and eReaders that may impact the reading experience. These elements are features of the eReading experience that frequently appear in comments by participants in related studies. We focus our research here on just one aspect of the eReading experience; how different facets of interface design for page-turning in mobile eReader software can impact readers success and preferences.

Page-turning in eReading studies can be addressed using either a hardware or a softwarebased solution depending on the device used for reading. This research looks specifically at software-based page-turning solutions. A case study investigation of current eBook page-turning norms in readily available eReader software was undertaken, followed by a preliminary user study of reader preference for the use of these identified page-turning norms. This research explores the importance of the physical book metaphor when turning a page in an eReader and which pageturning methods are preferred by readers.

The remainder of this paper is structured as follows: Section 2 discusses related work on turning pages in printed and digital books. Section 3 audits the interface design and features of 10
eReader software across three similarly sized tablet devices. Section 4 explains the process that we followed to develop test material for this investigation, and the method of our page-turning user observation and interview study. Section 5 reports the study results of this investigation. The paper concludes with Sections 6 and 7 discussing the results and contributions of our research, along with proposed future work in the area of pageturning for eReader software interfaces.

\section{RELATED WORK}

Despite many differences between the two media, expectations from experience with physical books (pBooks) are carried over to the eReader experience (Wilson, Landoni \& Gibb, 2002). When moving through the pages of a book readers may do everything from turning single pages slowly as they read, through to rapidly flipping through multiple pages. Turning pages is an essential aspect of navigating a book but the reason why the reader is navigating that book can change the way and speed with which they turn the pages (Marshall, 2010). For example, turning the pages of a novel is very different to skim-reading a text for a specific snippet of information that is required. The implementation of page-turning in a digital book is a navigation function which has the ability to enhance (Wilson, 2001), and equally could negatively impact the eReading experience.

While hardware page-turning investigations have been sparse, to-date no research has compared software-based page-turning systems. Investigations regarding eBooks, eReaders, and 
eReading is becoming dated, with very little investigation into reading on modern mobile devices such as smartphones and touch screen tablet devices. At this point, there remains little evidence to support which interactive functions for page-turning in eReaders are preferred by readers.

\subsection{Page-turning in Physical Books}

Turning pages in a physical book is fast and intuitive, with many readers anticipating the page turn by lifting the corner of the page before they are ready to turn the page (O'Hara \& Sellen, 1997). These 'light-weight' interactions involved in turning a page do not simply involve the act of turning the page but involve pre-emptive actions before the page is turned (Marshall \& Bly, 2005). Tajika et al. (2008) observed three main types of page-turning when navigating physical pages. These were; turning a single page at a time, flipping through many individual pages at once and "leafing" which they described as turning several pages at once. Each of these page-turning operations were conducted in a range of ways by individual participants. This small study showed the breadth of ways that users intuitively interact with physical books and the range of page-turning methods that would need to be supported by eReaders to achieve similar levels of intuitive use by readers.

Further to this, Kim, Kim \& Lee (2013) showed that the flexible, manual nature of paper enables readers to navigate easily between pages of a print book. Conversely, buttons and scroll bars provided in eReaders do not provide this full range of functionality to facilitate all of the actions conducted by readers of paper documents, and when they are included can take up valuable screen space (Kim, Kim \& Lee, 2013), especially on eReading devices with smaller screen sizes.

\subsection{Page-turning in eReader Hardware}

Page-turning functions in electronic books can vary greatly depending on the physical device and software being used. A wide range of both hardware and software solutions have been implemented in different products (Marshall, 2010). Here we discuss briefly the related work for pageturning on eReader hardware which appears to be more thoroughly investigated to date than eReader software solutions for page-turning.

Several studies have looked broadly at the usability of different eReader devices and the preferences of readers (Wilson, 2003; Gibson \& Gibb, 2011; Richardson \& Mahmood, 2012), yet none of these have specifically focused on page-turning and its function within the reading experience.

The most common method for turning pages in dedicated eReader hardware is through the use of buttons, while lesser used hardware supported navigation methods include rocker switches and sensors (Marshall, 2010). These buttons and dials which are physically placed outside of the software environment, as features of hardware devices, should be developed to ensure smooth and fast control of the eBook. Page transitions and insoftware features must be perceived by the user to be rapid and reactive to the users input. For example, Wilson et al. (2002) showed the importance of the buttons in these systems being large enough to turn pages easily in both directions. In investigations by Wilson (2003), participants felt the dial on the Jornada device was fast for turning pages. Page-turning in the Jornada was preferred over paper by some participants in that study. Research by Behler (2009) with the Sony PRS-505 model of eReader noted that participants found that it had a slow screen refresh when page-turning and this was frustrating for many of the participants. This observation relates to the theory discussed by Marshall and Bly (2005) that when turning a digital page, the reader can lose the visual connection with the text briefly.

Whether in hardware or software the buttons for page-turning should be conveniently placed (Wilson, 2001). The speed at which the digital page refreshes or turns has been found to be an important factor for readers on digital devices, and the 'flash' that occurs when turning pages on elnk devices is negatively perceived by readers (Richardson \& Mahmood, 2012; Behler, 2009; Gibson \& Gibb, 2011).

Further research into hardware solutions for pageturning has looked at providing solutions which have a stronger connection between how we would navigate a physical book, and page-turning in eBooks. Researchers have investigated the potential of flexible reading surfaces to assist readers with mimicking the range of page-turning approaches that are often used in physical book reading situations (Wightman, Ginn and Vertegaal, 2010; Tajika, Yonezawa \& Mitsunaga, 2008).

\subsection{Page-turning in eReader Software}

Dedicated eReaders, especially elnk readers, have both advantages and disadvantages over devices where reading eBooks is one of their many functions. Limitations noted regarding elnk readers have included format restrictions; text, image, and colour rendering limitations; shortcomings with physical interaction methods; and size and weight implications (Allmang \& Bruss, 2010; Gibson \& Gibb, 2011). Using non-dedicated devices for eReading may avoid some of these issues. Here we report the sparse work related to page-turing in eReader software.

Marshall (2010) and Chu et al. (2004) discuss the relationship of eReader page-turning to physical book page-turning. When eReader interfaces strongly replicate or pay homage to the pBook 
page-turning metaphor it can be argued that the physical act of turning pages is emphasised as being important to the reading experience. Conversely, when page-turning functions do not mimic the pBook usability, speed, and functionality are afforded greater emphasis in the eReader.

The Realistic Books software (Liesaputra \& Witten, 2012), mimics physical page-turning to discover if the realistic simulation provides benefits to readers over other page-turning methods. This research found that the simulated page-turning animation was more engaging for the reader. The 'Bezelflipper' solution developed by Kim, Kim \& Lee (2013), is a software-based solution to allow readers of eBooks to flip single or multiple pages on a multi-touch tablet based on a paper book metaphor. Their evaluation showed the prototype to be well received by participants. Participants required tutorship on the use of their system before correctly and intuitively using their proposed pageturning metaphor showing a need for visual cues in eReading software to assist with page-turning.

\section{EREADER PAGE-TURNING CASE STUDY}

As we have shown in the related work little previous research has analysed page-turning preference for modern eReaders, especially in software. To develop a comprehensive picture of the current state of page-turning methods we conducted an audit of a wide range of the available eReader technologies at the time of this study. With eReader software available on modern mobile devices we investigated eReader software on the Android, and iOS mobile device platforms and compared this to a dedicated elnk eReader hardware device-a Kindle KPW2. To ensure comparability, we reviewed available eReader apps on a Samsung Galaxy Note 8.0 tablet (Android), and an iPad Air 2 (iOS) tablet in our study as these were similarly sized devices to the Kindle eReader and were readily available on the market at the time of this study. The Kindle KPW2 is a touchscreen device where the page-turning is integrated into the screen rather than being a physical feature of the hardware. These three devices were selected in order to review how pageturning and interactive elements work for applications across typical eReading systems at the time of the study.

To identify an appropriate cross-section of eReader apps to review the search term "popular eBook apps" was used on the Google search engine. We identified ten applications to be studied, five of which were able to be compared across both Android and iOS (see Table 1). In total, we reviewed seven apps on Android and eight on iOS. Unsurprisingly, because the Kindle is an elnk device it does not allow for non-native eReading apps to be installed and therefore only the native Kindle eReader was considered on this device. The Kindle app is available for Android and iOS and therefore was a feature of our study on all three platforms.

Table 1: eReading apps tested

\begin{tabular}{|l|c|c|c|}
\hline \multirow{2}{*}{ eReader Apps } & \multicolumn{3}{|c|}{ eReading Devices } \\
\hline & Android & iOS & Kindle \\
\hline Kindle & $\checkmark$ & $\checkmark$ & $\checkmark$ \\
\hline Blio & $\checkmark$ & $\checkmark$ & \\
\hline Bluefire & $\checkmark$ & $\checkmark$ & \\
\hline Kobo & $\checkmark$ & $\checkmark$ & \\
\hline eBrary & $\checkmark$ & $\checkmark$ & \\
\hline BookReader & $\checkmark$ & & \\
\hline Txtr eBooks & $\checkmark$ & & \\
\hline iBooks & & $\checkmark$ & \\
\hline Marvin & & $\checkmark$ & \\
\hline Megareader & & $\checkmark$ & \\
\hline
\end{tabular}

Each app was installed on the appropriate tablet device and an eBook was opened in each application so that the interactive features and page-turning devices for each app could be audited. We identified the page-turning functions that were available in each of the apps and then classified them into six page-turn methods. We refer to these six methods as; Arrows, Swipe, Page Curl, Tap/Touch, Slider, and Page MiniView. Table 2 details the availability of each of these identified page-turning methods for each of the tested apps. All of the apps reviewed implemented at least two ways to turn pages and provided visual cues in the form of animation, interactive icon, or movement to assist the reader to understand how to use the page-turning features of the software.

The methods of page-turning ranged from those which were a direct metaphor of page-turning in a physical book, through to abstract page-turning navigation using buttons or touch points. Arrows and Tap/Touch are both page-turning methods that are abstracted from the related action in a pBook, while the Swipe and Page Curl methods are a metaphor of the equivalent interaction in a pBook. The slider and Page MiniView are methods that incorporate page-turning with orientation cues for the reader, meaning that orientation cue features were often separate from the page-turning feature if they were present in the eReader app. Arrow, Swipe, and Tap/Touch were the most commonly identified page-turning techniques in the apps that we audited. 
Table 2: Page-turning features of eReading apps tested

\begin{tabular}{|c|c|c|c|c|c|c|}
\hline App & 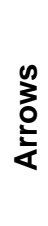 & $\stackrel{\frac{2}{3}}{\frac{0}{3}}$ & 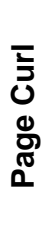 & 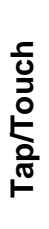 & $\frac{\grave{0}}{\frac{0}{\sigma}}$ & $\frac{3}{\stackrel{3}{0}}$ \\
\hline Kindle (Android) & $x$ & $r$ & $x$ & $\checkmark$ & $\checkmark$ & $\checkmark$ \\
\hline Kindle (iOS) & $x$ & $\checkmark$ & $x$ & $\checkmark$ & $\checkmark$ & $x$ \\
\hline Kindle (Kindle) & $\checkmark$ & $\checkmark$ & $x$ & $\checkmark$ & $x$ & $x$ \\
\hline Blio (Android) & $\checkmark$ & $\checkmark$ & $\checkmark$ & $\checkmark$ & $\checkmark$ & $x$ \\
\hline Blio (iOS) & $x$ & $\checkmark$ & $r$ & $\checkmark$ & $x$ & $x$ \\
\hline Bluefire (Android) & $\checkmark$ & $\checkmark$ & $x$ & $x$ & $\checkmark$ & $x$ \\
\hline Bluefire (iOS) & $\checkmark$ & $\checkmark$ & $x$ & $\checkmark$ & $\checkmark$ & $x$ \\
\hline Kobo (Android) & $\checkmark$ & $\checkmark$ & $x$ & $\checkmark$ & $\checkmark$ & $\checkmark$ \\
\hline Kobo (iOS) & $x$ & $r$ & $x$ & $\checkmark$ & $\checkmark$ & $x$ \\
\hline eBrary (Android) & $x$ & $\checkmark$ & $x$ & $\checkmark$ & $x$ & $x$ \\
\hline eBrary (iOS) & $x$ & $\checkmark$ & $x$ & $\checkmark$ & $x$ & $x$ \\
\hline Book Reader (Android) & $\checkmark$ & $\checkmark$ & $x$ & $\checkmark$ & $x$ & $x$ \\
\hline Txtr eBooks (Android) & $x$ & $\checkmark$ & $x$ & $\checkmark$ & $x$ & $x$ \\
\hline iBooks (iOS) & $\checkmark$ & $\checkmark$ & $x$ & $\checkmark$ & $x$ & $x$ \\
\hline Marvin (iOS) & $\checkmark$ & $\checkmark$ & $x$ & $\checkmark$ & $\checkmark$ & $x$ \\
\hline Megareader (iOS) & $\checkmark$ & $\checkmark$ & $x$ & $\checkmark$ & $x$ & $x$ \\
\hline
\end{tabular}

Arrows was usually implemented as left and right arrows or triangle shaped buttons at the foot of a page and advanced or regressed one page at a time. Nine of the 16 apps audited incorporated the Arrows page-turning method.

The Swipe feature was used by all of the apps that we investigated where the swipe of a finger across the screen to the left would advance the page and to the right would regress the page. Of the pageturning methods found in this audit, this swipe method is perhaps the most synonymous with page-turning in a physical book. We identified that Blio on both Android and iOS used a PageCurl effect when swiping. This perhaps increases the physical book metaphor further than the simple slide or page advancement present when a finger swipe is made by the user.

The Tap/Touch page-turning method was used in 15 of the apps investigated. When a user tapped towards the right-hand side of the app the book would advance by a single page and when the reader tapped on the left-hand side of the app a single page regression occurred.
Eight of the eReader applications had a Slider at the bottom of the screen, allowing readers to drag the Slider to change chapters or pages. The Slider typically presented as a progress bar with a fill that showed progress in the chapter or the book and a dragable interactive dot which could be used to advance or regress a single page or several pages.

Two of the eReaders incorporated what we have termed the Page MiniView system. This function showed an overview of the page currently being read, which was situated in the centre of the list of pages, and a small selection of the pages surrounding. Users were able to tap on any of the visible pages to advance or regress a single page or several pages at once.

The Kindle (KPW2) is a dedicated hardware device for reading eBooks. On this device readers could Swipe, tap or touch the screen to turn the page, and enlarged the screen to change the size of font.

As dedicated eReading hardware for reading eBooks and as an elnk device, the Kindle can only implement relatively simple page-turning methods such as use of the Arrows, Swipe, Tap/Touch to turn pages. With this technology, it would currently be difficult to implement the animated methods such as the Page Curl and Slider methods, however, the Page MiniView technique would be possible given the capabilities of the technology. This suggests that understanding user preference for all of these six page-turning methods warrants further investigation.

\section{PAGE-TURNING INTERFACE DESIGN}

To investigate user preference for page-turning methods we developed a user study that tested these six page-turning norms that were identified currently being implemented by eReader software.

We showed through our case study (see Section 3) that all of the eReaders audited allowed the user to turn the page using more than one method. While this is a sound approach for commercially available software to allow individual users to determine how they would like to interact with the software this did not allow for controlled scrutiny of page-turning methods during our study. To control the testing of each page-turning method we developed a test environment that allowed for reading of an eBook. In this test eBook the user was provided with one page-turning method at a time during the reading experiment. The test software implemented a different page-turning method per chapter and before a participant used each page-turning method they were presented with an in-app instructional video to demonstrate how each pageturning method functioned. 


\subsection{Method}

Participants were provided with the eReader test software on a Nexus 7 tablet. This software contained six chapters of a book each of which allowed the user to interact using a different pageturning method. When the user began working with the tablet they were presented with a welcome page so that no interaction began without the users explicit control and focus. Once the reader proceeded from the welcome screen they were presented an initial instructional video detailing the use of the first page-turning method that they would use to navigate the first chapter of the book. At the beginning of each page-turning method the reader was presented with an instructional video for that method. At the end of the instructional video the user was invited to read a few pages while using the interaction method that had been shown. Once the reader completed the reading task for an interaction method, an end of task screen was shown to the reader before they proceed to the next chapter and page-turning method.

Participants were encouraged to read, rather than simply interact with the device in order to immerse themselves in the reading experience as much as possible for a lab-based study such as this.

After the user had interacted with all six pageturning methods each participant partook in a short interview conducted by a single researcher. We sought basic demographic information as well as detail about the level of experience each reader had with digital reading. Participants used a Likert scale to describe how easy/hard they felt each page-turning method was. We also asked users to describe their reasons for selecting the Likert score for each page-turning method. We then asked six questions to understand their preferences; their sense of ease, their perception of the interaction method's intuitiveness, accuracy, and appropriateness for academic or pleasure reading.

\subsection{Participants}

Thirty individuals were recruited to participate in the investigation; all of them had a tertiary qualification and were 18 years or older. Our study included 15 participants between 18-25 years old, 8 participants between 26-35 years old, 3 participants between 36-45 years old, and 4 participants over 46 years old. All participants had experience reading eBooks on either a mobile phone or tablet device or an elnk device. 27 of our participants had experience reading academic eBooks or eArticles and only three participants reported that they had never read an electronic academic text. 21 participants responded that they had experience reading for pleasure in an electronic format.

\subsection{Design of Page-turning Methods}

The six page-turning methods that we tested were Arrows, Page Curl, Tap/Touch, Slider, Orientation Cue, and Page MiniView. As noted in the Method, these six page-turning methods were implemented as individual chapters in an Android app developed specifically for our testing requirements.

We will describe the design and implementation of each of these page-turning methods in this section. Example screens of the six methods can be seen in Figure 1. The Arrows and Tap/Touch methods were abstracted page-turning methods with the action having no physical relationship to pageturning in pBooks. The Slider, Orientation Cue and Page MiniView methods we tested all incorporated orientation cues into the page-turning function in different ways. Page Curl was the only pageturning method we tested that was a metaphor of the same action in a pBook.

\subsubsection{Arrows Page-turning Method}

Perhaps the most rudimentary method to be tested was the single-click left and right Arrows method. This approach was similar to a page-turning approach on the Kindle device. In both our devised Arrows method, and on the Kindle device in the Case Study, readers could click the arrow shaped buttons to turn the page. The right arrow advances a page and the left arrow regresses a page. As can be seen in the left most screen in Figure 1, the Arrows were placed at the bottom right with the page number directly below the interaction point.
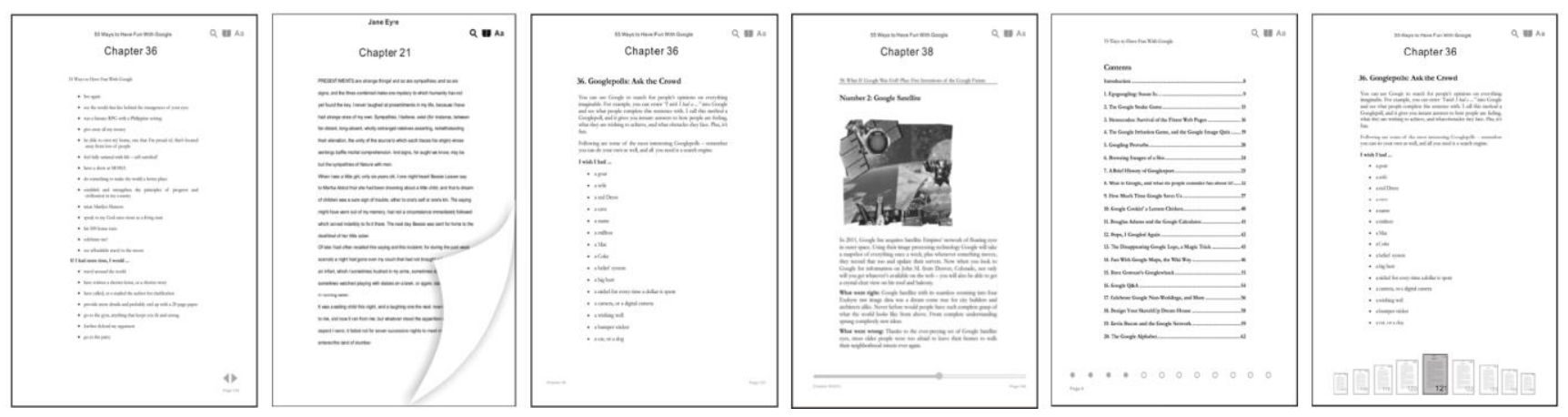

Figure 1: The six page-turning methods (I-r); 'Arrow', 'Page Curl', 'Tap/Touch', 'Slider', 'Orientation Cue', and 'Page MiniView'. 


\subsubsection{Swipe with Page Curl Page-turning Method}

Similar to the method seen in the Kobo in the Android system, the reader could Swipe the screen from right to left side to turn the page. In our implementation, a page-curl animation while the page is being turned visually imitates a physical page turn. Page numbering was at the bottom right of the page. Our Page Curl screen is shown as the second screen from the left in Figure 1.

\subsubsection{Tap/Touch Page-turning Method}

The Tap/Touch method was implemented such that when the reader touched the screen, the page was turned. Participants tapped the right side of the screen to advance the page and tapped the left side of the screen to regress a page. Page numbers were provided on the bottom right of the page (see the third screen from the left of Figure 1).

\subsubsection{Slider Page-turning Method}

Similar to Kindle, Bluefire, Blio and Kobo in the Android system and Kindle, Bluefire, Kobo, Marvin in the iOS system; the Slider page-turning method allowed readers to drag the dot along the bar to turn the page. This page turning method is shown as the third screen from the right in Figure 1. Each page corresponded to an equal length on the Slider. At the bottom left of the page it showed the reader the number of the chapter and what percentage they had read, the bottom right side showed them the page number.

\subsubsection{Orientation Cue Page-turning Method}

Each dot at the bottom of the page represented a single page. The reader could tap the dot to turn the page, or just click any dot to jump to any page. In the example for this study 12 dots were present at the bottom of the page, but this can be altered depending on the number of pages in a book chapter. This page turning method is shown as the second screen from the right in Figure 1. This method was different from the sliders seen in the case study, this method allowed for a tap rather than a drag to turn pages. This method of turning pages was not seen in the case study and was devised based on extension of the Slider method and interpretation of the research of Vanderschantz et al. (2017).

\subsubsection{Page MiniView Page-turning Method}

In this method, the foot of the page in the eReader was dedicated to the Page MiniView which consisted of nine miniature representations of the eBook's pages. The page in the middle, which was also the largest and highlighted, represented the current page. Participants could tap the page to the right of the highlighted page to advance, or the page to the left to regress. Additionally, participants were able to tap any of the visible pages in the MiniView to move directly to that page. When participants click the page, the current page shifted to the centre and became enlarged and highlighted. Each page of the MiniView had a page number. This page turning method is the right most screen in Figure 1.

\section{RESULTS}

Here we discuss the results of our interviews following the reading observation.

\subsection{Perception of Ranked Ease of Use}

After completing the reading exercise, the interview began by asking participants for their perception of the ease of use for each of the six page-turning methods. The participants were asked to rank the ease of use of each of the methods that they had interacted with based on a 5-point Likert scale (from 'very easy through to 'very hard'). We report the results of this Likert scale question in Table 3 and visualise this data in Figure 2.

Table 3: Ease of use Likert results ( $n=30)$

\begin{tabular}{|c|c|c|c|c|c|}
\hline & 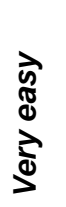 & 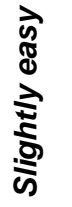 & 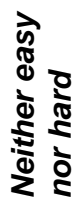 & 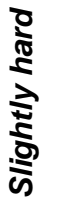 & $\frac{\sqrt{2}}{\frac{\pi}{2}}$ \\
\hline $\begin{array}{l}\text { Orientation } \\
\text { Cue }\end{array}$ & 13 & 8 & 4 & 5 & 0 \\
\hline Arrows & 13 & 10 & 2 & 3 & 2 \\
\hline Swipe & 15 & 3 & 7 & 5 & 0 \\
\hline Tap/Touch & 25 & 2 & 1 & 2 & 0 \\
\hline Slider & 6 & 4 & 4 & 15 & 1 \\
\hline MiniView & 15 & 10 & 2 & 3 & 0 \\
\hline
\end{tabular}

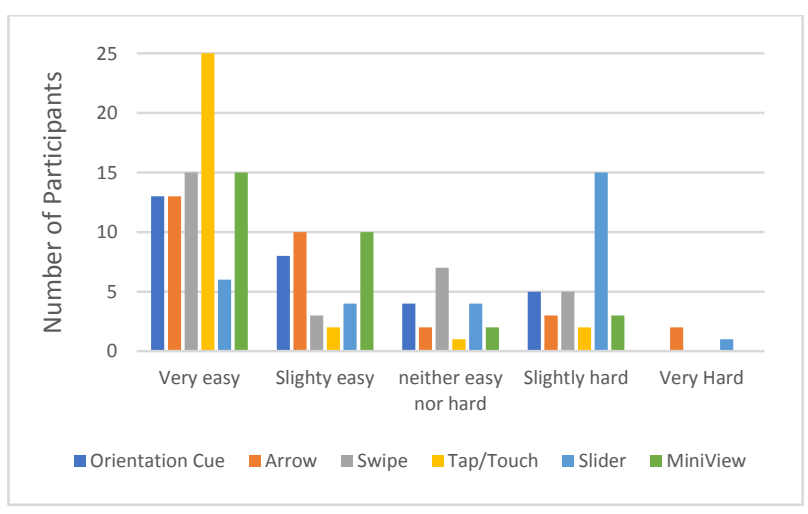

Figure 2: Ease of use Likert results $(n=30)$

Overall it can be seen that the majority of participants felt that all of the page-turning methods were either 'very easy' or 'easy' to use, other than the Slider page-turning method. Tap/Touch was the page-turning method that was most commonly 
thought to be 'very easy' to use with 25 participants responding that Tap/Touch was very easy to use.

Responses for Arrows were closely divided between 'very easy' (13) and 'easy' (10), as was the case with the responses to Page MiniView with 15 responding that it was 'very easy' and 10 responding that it was 'easy'. A similar spread of responses was seen for Orientation Cue with 13 thinking it was 'very easy' to use and 8 feeling that it was 'easy' to use.

For Swipe 'very easy' (15) was the most common response, but the next greatest number of responses for Swipe was that it was 'neither easy nor hard' (7).

The most common response for the Slider was that it was 'hard' with 15 participants responding that way, and one participant felt that it was 'very hard'.

\subsection{Comparative Perception of Ease, Preference, Accuracy, Intuitiveness, Appropriateness}

The second stage of the interview process asked participants to directly compare each of the six page-turning methods in regard to which was; easiest to use, most preferred, most accurate, most intuitive and most appropriate for turning pages. Participants were asked to provide reasons for their decisions. We report these results here.

\subsubsection{Comparative Perception of Ease}

Participant's responses to the question 'Which approach was the easiest to turn pages?' (Figure 3) showed that the Tap/Touch method was perceived as being the easiest page-turning method. Tap/Touch was considered easiest to use by 14 participants, with 12 of them describing this approach as "fast", "efficient" or "time saving". The remaining two participants who felt that Tap/Touch was the easiest method described this as a "convenient" method because they did not need to find a specific position to click.

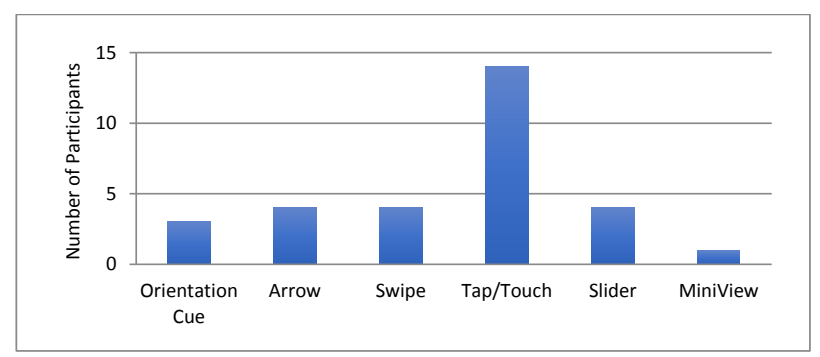

Figure 3: Comparative perception of ease $(n=30)$

Three participants chose the Orientation Cue as the easiest, while four participants chose the Arrows because they thought these approaches were intuitive. Four participants considered Swipe easy because they considered this method to be similar to swiping or turning pages in pBooks.
Finally, four participants thought the Slider was very easy, particularly for jumping to pages further than one page before or after and only one participant described Page MiniView as easiest believing that the page overview was intuitive and informative because it provided page numbers and page overviews.

This result mirrors the initial ranking (see Section 5.1) that showed $25 / 30$ participants identifying Tap/Touch to be "very easy" to use. The difference with this question is that we asked participants to identify the one page-turning method that they perceived to be the easiest for turning pages.

\subsubsection{Comparative Preference}

We asked participants 'Which approach did you like best to turn pages?' (Figure 4). 15 participants liked the Tap/Touch method the most, these participants thought this approach was fast to turn pages and provided the participants with the ability to turn pages without needing to click a specific interface position. Six participants chose Page MiniView, because they could preview the page content and page number, which was described as convenient for finding information. Six participants chose Swipe as their favourite approach to turning the page, which they thought was similar to reading a pBook. There were only two participants who preferred the Slider to turn the page; these two participants considered this approach to be more accurate to turn pages than the other methods and described this as their reason for this preference. Only one participant described Orientation Cue to be the method that they like the most. No participants selected the Arrow as the most preferred method to turn the page; many participants thought the arrows were too small to touch accurately.

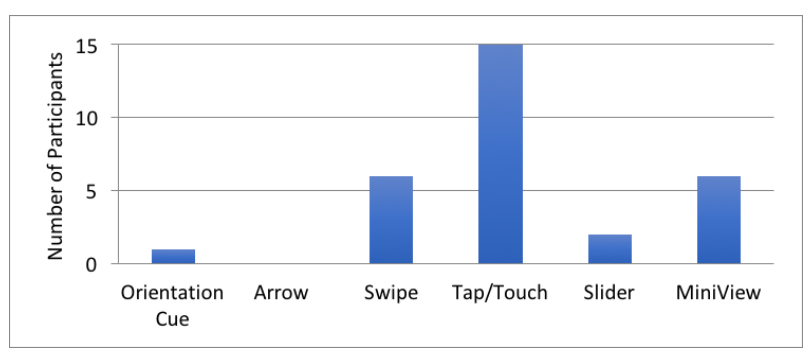

Figure 4: Comparative preference $(n=30)$

\subsubsection{Comparative Perception of Accuracy}

When asked 'Which approach was the most accurate to turn pages?' (Figure 5) the perception of accuracy was more widely spread amongst the methods that we tested, compared to the perception of ease of use or preference. Nine participants described the Page MiniView method as intuitive, with 7 of 9 specifically describing the ease of scanning the individual page numbers presented on the miniature pages as assisting with 
accuracy. Three participants thought the Orientation Cue method was the most accurate approach for turning pages, because it was intuitive and easy to touch the dots. Six participants chose the Arrows, with three people specifically describing the ease of following the arrows direction for an indication of progressing or regressing the book. Four participants chose Swipe, because they thought it was like a real book, so they knew how to use this approach quickly. Eight participants chose Tap/Touch, because this approach could turn pages one by one. None of the participants chose the Slider as the most accurate approach.

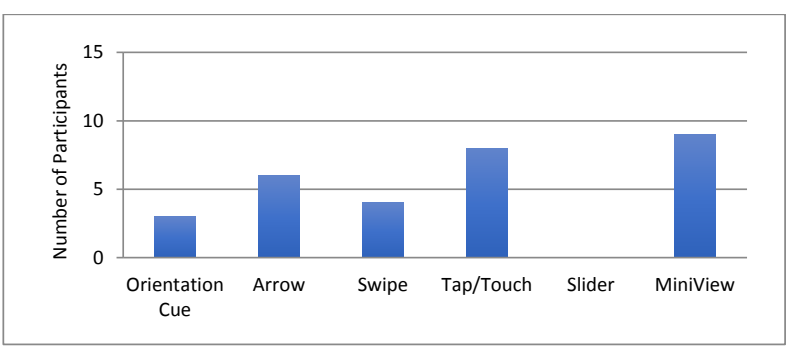

Figure 5: Comparative perception of accuracy $(n=30)$

\subsubsection{Comparative Perception of Intuitiveness}

Similar to sense of accuracy, when asked 'Which approach was the most intuitive to turn pages?' (Figure 6) participants most commonly responded with Page MiniView. 13 participants chose Page MiniView because they could preview the page and easily identify the specific page or page number they wanted to navigate to next. Seven participants chose the Arrows as the most intuitive approach, two people described this approach as "straightforward" and five participants again discussed the directionality of the arrows as assisting with navigation. Five participants chose Swipe, because it was like a pBook. Three participants chose Tap/Touch reporting that they did not need to take time to think how to interact with the device. Two participants chose Slider stating that it was easy to understand and use. None of the participants chose the Orientation Cue method as the most intuitive method.

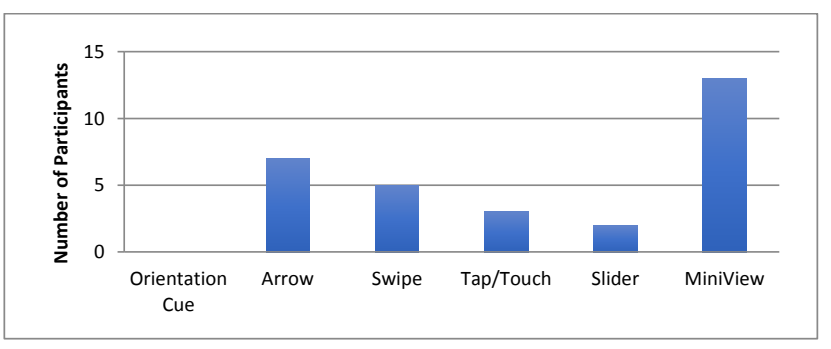

Figure 6: Comparative perception of intuitiveness $(n=30)$

\subsubsection{Comparative Perception of Appropriateness}

Finally, we asked participants to consider the pageturning methods and which method they felt was more appropriate for academic information reading and in a separate question, which approach they felt was more appropriate for pleasure reading. The first of these two questions were worded 'Do you think one of these approaches is more appropriate for academic information reading?' and after answering that question the participant was then asked 'Do you think one of these approaches is more appropriate for pleasure reading?' (Figure 7).

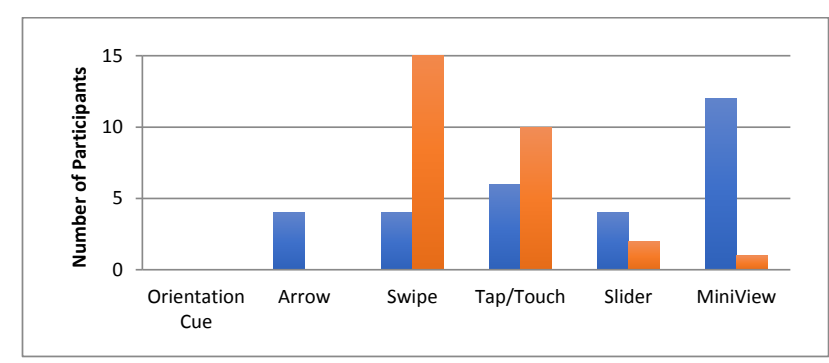

Figure 7: Comparative perception of appropriateness for academic and pleasure reading $(n=30)$

For an academic reading situation twelve participants chose Page MiniView because readers could preview the page content and page numbers, which saved time when searching for useful information. Two participants discussed that this approach provided an easy way to skip pages which would be useful in academic reading.

Six participants chose Tap/Touch because it was fast to turn pages. For academic reading, the readers did not want to feel like they were "wasting time on waiting for the page to "turn" and thought it would be helpful for finding information quickly.

Four participants chose the Slider because it was easy to touch and provided an easy way to move along sections of the book or chapter.

Arrows was chosen by four participants, simply because it was easy to understand in any reading situation.

Four participants chose swipe because this approach was similar to real books, so readers could adapt to use this approach quickly.

None of the participants thought the Orientation Cue method was the most appropriate page-turning method for academic reading.

For pleasure reading participants' choices and reasons differed to their answers for academic reading. Swipe was chosen the most frequently, by 17 participants. The reasons given by these participants were because it was similar to reading real books, readers enjoyed the page-turning animation and slow reading experience.

Ten Participants felt the Tap/Touch page-turning approach was most appropriate for pleasure reading because it was fast to turn the page. A common description was that if they could not wait to read the next page or chapter in an exciting 
novel then they could quickly turn to that next page or chapter of the "page turner" they are reading.

The two participants who chose Slider did so because it was interesting. One participant thought the Page MiniView approach was appropriate for pleasure reading because readers could preview the content and page numbers in the MiniView.

No participants chose either the Orientation Cue or the Arrows method as the most appropriate pageturning method for pleasure reading.

\section{DISCUSSION}

In the case study, we identified a range of pageturning methods that might influence the experience of readers. We then developed six page-turning approaches based on the case study that were tested and discussed by 30 readers. We reviewed participants' preference for ease, intuitiveness and accuracy of the six approaches. Participants gave various reasons for their preference for the page-turning methods studied, yet three key factors that affected their preferences emerged: 1) the speed of interaction with a pageturning method; 2) the size of interactive points; and 3) the ease of utilization of a method.

The two approaches that received the most discussion by participants and ranked most highly for ease of use were the Tap/Touch and Page MiniView approach. Tap/Touch method featured highly when it came to sense of ease and user preference. The Page MiniView method featured highly in the investigation into accuracy, intuitiveness and appropriateness, while both of these methods were deemed to increase speed and efficiency in navigating the book.

Participants who preferred Tap/Touch and MiniView gave similar reasons why they chose this approach. Most participants discussed these two approaches as convenient for page-turning and the page-turning speed was considered appropriate, if not fast. This supports previous research where it was found that readers were frustrated by slow page-turning (Richardson \& Mahmood, 2012; Behler, 2009; Gibson \& Gibb, 2011) or buttons or dials not being in an intuitive position (Wilson, 2001). Our participants also considered the Tap/Touch approach easy to use and understand - they could touch the screen without hesitation and they did not have the feeling that they were "wasting time thinking about clicking" on a specific position to turn the page.

As often occurs in studies of eBooks, user's familiarity and affinity with traditional pBooks was a factor in this study. It was also found that users' expectations from eBooks are inherited from their experience with pBooks. The reason is pBooks increase users' subjective satisfaction (Malama,
Landoni \& Wilson, 2005; Landoni, Wilson \& Gibb, 2000). All of our participants were familiar with reading both eBooks and pBooks and all participants at some point during their interview discussed their preference for a particular pageturning method because it was intuitive or familiar. Examples of this were when users discussed Tap/Touch, PageCurl, Arrows, and Sliders. All of these page-turning methods are similar to a pBook metaphor or another common digital interaction and therefore have strong familiarity for the user.

Users in our study stated that they liked the feeling of swiping the screen to turn pages because they thought the animation of page-turning was similar to holding a pBook in their hands. It is possible that participants in our study showed a preference for the approach that they could understand immediately, or they had previously encountered in other eBook applications or physical book reading experiences. We also identified participants that felt the pBook metaphor is not necessarily important for readers. These participants often prioritised ease of use and intuitive interaction.

\subsection{Negative Perceptions \& Future Work}

We did not seek to specifically investigate negative views of the interaction methods that we studied. For this reason, very little data is available to report the perceived negatives of any of the interaction methods. Participants did note when describing why they chose a particular method that size of interactive devices and ease of interaction was important to them when reporting their preferences.

Most participants considered the Arrows method was easy to understand, however the size of the Arrows was described as being too small or too close to each other by some participants. Similarly, the Orientation Cue method was considered difficult by some users due to the placement of dots appearing close to other dots which concerned users about their ability to accurately select the page they wished to navigate to. Interestingly, while orientation cues (Marshall, 2010) were not a central feature of this study, this method was not ranked highly by participants for page-turning, yet similar methods by Vanderschantz et al. (2017) were ranked highly for orientating in eBooks and pBooks. Investigations that consider if and how a single method might facilitate orientation as well as pageturning is warranted based on this observation.

There were only a few participants who preferred or considered the Slider the easiest page-turning method; many participants found this approach frustrating, because they struggled with the refined need to touch the slider in a particular manner to gain the result they wanted. This method and the Page MiniView method did however allow for moving in chunks or progressing or regressing by more than one page at a time. The slider is 
perhaps the more typical page-turn method of the two which allowed for this type of multi-page advancement yet ranked lowly in our study. Conversely Marshall \& Bly (2005) discussed that being able to turn multiple pages at once was important for readers based on their studies.

Speed was also noted as important, particularly when describing the Touch/Tap method as being superior because the reader could touch anywhere to the left or the right of the screen to achieve a result. Related to this, it was noted in discussion by some participants that the Page Curl and the Swipe methods appeared slow compared to other methods. From this that it can be inferred that the physicality and the perceived accuracy and speed of a page-turning navigation device also contributes to the negative perceptions of these page-turning methods. Future work would do well to specifically investigate the negative perceptions associated with any or all of these page-turning methods.

\subsection{Screen Real-estate}

Page MiniView was a favoured page-turning method however this method has the potential to divide user preference as it hinders the amount of content possible per page. Kim, Kim \& Lee (2013) noted some methods of button or scroll bar for page-turning in eReaders can take up valuable screen space for smaller devices and alluded to this as a limitation of page-turning features such as scroll bars and software buttons.

\subsection{Qualitative Insight for Designers}

The feedback provided by participants also allows some insight into design considerations for pageturning in eBooks. According to some of our participants, a fast page-turn result is necessary for reading eBooks, because it would "save time" when reading. The sizing and spacing of interactive points received a significant amount of discussion from participants. Button sizing and positioning features highly in the interface design literature for mobile interfaces yet has not features significantly in the eBook or eReading literature to date.

Some participants considered it was important to have an introduction or navigation guide at the start of an eBook, in order to help people new to digital reading to understand the novel use of an eBook quickly. We hypothesise that this may be most relevant for an older generation of reader and may prove less necessary as the greater population becomes more familiar with daily touch screen interaction metaphors.

\section{CONCLUSION}

This paper identified and tested six different pageturning approaches and considered the preferences of users. The six approaches that were identified and tested included the use of Icons, Arrows, Swipe, Tap/Touch, Sliders and Page MiniViews. It was found that different interactive elements of page-turning effected readers' preferences differently based on their reading need or purpose. In-line with the conclusion of Marshall (2010), it seems that we have also shown that "it depends!" (p.52).

Based on the results of the research conducted for this investigation, we summarise here advice for publishers and designers based on the evidence of testing of six page-turning approaches for eBooks. Vital elements for designing a successful eBook are; the speed of page-turning, the intuitiveness of page numbering and the suitable size of interactive elements all play key roles in an eBook.

\subsection{Recommendations}

This study indicates that where possible pageturning devices that allow for rapid individual page advancement or regression are required as well as allowing users to advance and regress multiple pages quickly. Our findings suggest that Tap/touch methods may prove successful for continuous and linear reading as is often typical of fiction texts and texts that require a student or academic to read deeply. Similarly, the Swipe approach was shown to be preferable for pleasure reading because it provided for an intuitive and relaxing experience. For reading situations that require scanning and assessing content that is one to several pages from the current page methods such as the Page MiniView may present students and academics with suitable mechanisms for non-linear and nonfiction searching and reading.

In all of the commercial eReaders that we audited for this research users were provided with multiple methods for turning pages and navigating eBooks. With these different page-turning features providing different advantages in different reading situations, perhaps it would be useful to provide users more than one page-turning method to choose from in a single eBook application.

Speed of interaction and responsiveness of the eReader was also a factor of this study and requires consideration by manufacturers to ensure uptake and continued use of systems. As was shown by the eReader audit, this is particularly relevant because a reader has a plethora of choice of apps to read their eBook on when reading on an Android or iOS mobile device.

We have investigated solutions and preferences for page-turning in software, however, there are also many hardware solutions for page-turning. Further investigation needs to be conducted to understand whether hardware or software solutions for pageturning are preferred by readers. 


\section{REFERENCES}

Allmang, A. and Bruss, S. (2010). What customers want from kindle books. Online, http://www.nist.gov/nvl/upload/WhatCust omersWantFromKindlebooks.pdf p.36-9, Retrieved March 152017.

Behler, A. (2009), E-readers in action: an academic library teams with Sony to assess the technology, American Libraries, 40(10):56-9.

Chu, Y. C., Bainbridge, D., Jones, M., \& Witten, I. H. (2004, June). Realistic books: A bizarre homage to an obsolete medium?. In Proceedings of the 4th ACM/IEEE-CS joint conference on Digital libraries (pp. 78-86). ACM.

Gibson, C. and Gibb, F. (2011). An evaluation of second-generation ebook readers, The Electronic Library, 29(3):303-319.

Kim, S., Kim, J., \& Lee, S. (2013). Bezel-flipper: design of a light-weight flipping interface for ebooks. In CHI '13 Extended Abstracts on Human Factors in Computing Systems, pages 17191724. ACM.

Landoni, M., Wilson, r., and Gibb, F. (200). "From the Visual Book to the Web Book: The Importance of Design," The Electronic Library, vol. 18 , no. $6,2000$.

Liesaputra, V. and Witten, I. (2012) Realistic Electronic Books. International Journal of Human-Computer Studies, 70(9):588-610.

Malama, C., Landoni, M., and Wilson, R. (2005) "What Readers Want: A Study of Efiction Usability," DLib Magazine, vol. 11, no. 5, 2005.

Marshall, C. C. (2010). Reading and writing the electronic book. Synthesis lectures on information concepts, retrieval, and services, 1(1):1-185.

Marshall, C. C. and Bly, S. (2005). Turning the page on navigation. In Proceedings of the 5th ACM/IEEE-CS joint conference on Digital libraries, pages 225-234. ACM.
O'Hara, K. and Sellen, A. (1997). A Comparison of Reading Paper and On-Line Documents. Proc. CHI '97. pages 335-342, ACM Press.

Richardson, J.V. and Mahmood, K. (2012). eBook readers: user satisfaction and usability issues, Library Hi Tech, 30(1):170-185.

Tajika, T., Yonezawa, T. and Mitsunaga, N. (2008). Intuitive Page-turning Interface of E-books on Flexible E-paper based on User Studies. Proceedings of the 16th ACM international conference on Multimedia, October 26-31, 2008, pages 793-796. Vancouver, British Columbia, Canada.

Vanderschantz, N., Timpany, C., Hinze, A., Du, Y. (2017). Exploring Orientation Cues in Both Digital and Physical Books. Proceedings of the 31st International BCS Human Computer Interaction Conference. BCS.

Wilson, R. (2001). Evolution of Portable Electronic Books. Ariadne: Web Magazine for Information Professionals,

http://www.ariadne.ac.uk/issue29/wilson/ Retrieved March 152017.

Wilson, R. (2003). Ebook readers in higher education. Educational Technology and Society, 6(4):8-17.

Wilson, R., Landoni, M., and Gibb, F. (2002). A user-centred approach to e-book design. The Electronic Library, 20(4):322-330.

Wightman, D., Ginn, T., and Vertegaal, R. (2010). TouchMark: Flexible document navigation and bookmarking techniques for e-book readers. In Proceedings of Graphics Interface 2010, pages 241-244. Canadian Information Processing Society. 